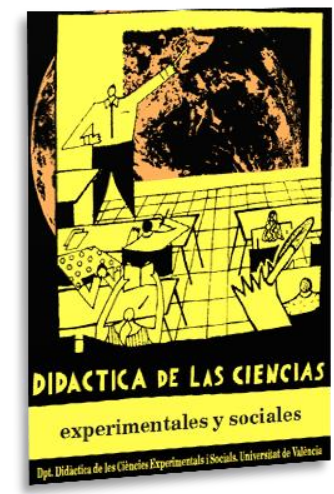

\title{
La metodología en el trabajo de huerto escolar y coherencia con la ambientalización curricular. Análisis de una práctica docente
}

\section{The methodology in the school garden work and coherence with curricular environmentalization. Analysis of a teaching practice}

DOI: $10.7203 / D C E S .35 .12799$

\author{
M. ${ }^{a}$ Carmen Conde Núñez \\ Universidad de Extremadura, cconde@unex.es \\ ORCID iD: https://orcid.org/0000-0003-1473-3531
}

Paula Mariscal Díaz

Universidad de Extremadura, paulamariscal94@gmail.com

\section{J. Samuel Sánchez Cepeda}

Universidad de Extremadura, samuel@unex.es

ORCID iD: https://orcid.org/0000-0001-8346-7236

\begin{abstract}
RESUMEN: Esta investigación presenta un estudio de casos de una maestra de Educación Primaria que trabaja con el huerto escolar. Su fin es conocer el grado de integración de la sostenibilidad en el proceso de enseñanza-aprendizaje y la metodología llevada a cabo con el huerto escolar. Se diseñan una serie de indicadores para cada una de las categorías de ambientalización curricular propuestas. Para analizar los resultados hemos utilizado una metodología cualitativa de corte interpretativo.
\end{abstract}

Palabras Clave: sostenibilidad, metodología de enseñanza, huerto escolar, estudio de caso, Educación Primaria.

ABSTRACT: This research introduces a case study of a primary school teacher who works with the school garden. It aims to know the degree of integration of sustainability in the teaching-learning process and the methodology carried out with the school garden. A series of indicators are designed for each of the proposed curricular background categories. In order to analyse the results we have used an interpretative qualitative methodology.

KEYWORDS: sustainability, teaching methodology, school garden, case study, Primary education.

Fecha de recepción: julio de 2018

Fecha de aceptación: octubre de 2018

Trabajo respaldado por el Proyecto de Investigación EDU2016-77007-R (AEI/FEDER, UE) del Ministerio de Economía y Competitividad de España. 


\section{INTRODUCCIÓN}

Hoy en día la situación ambiental en la que estamos inmersos ha hecho que muchas personas se replanteen su forma de vida y la manera de educar a las futuras generaciones desde la sostenibilidad, o incluso el decrecimiento, en el que ya estamos inmersos.

El huerto escolar es un modelo muy práctico donde se puede descubrir y aprender la estrecha relación que hay entre el hombre y la naturaleza, ya que nuestras raíces, están fijadas en la tierra y cada vez somos más conscientes de que somos integrantes de un ecosistema al cual estamos todos ligados dependiendo unos de otros, afirma Escutia (2009). Este modelo de huerto escolar ecológico pretende ser la base para el aprendizaje integrado mediante experiencias motivadoras y del mundo real (Eugenio y Aragón, 2016).

Se pretende con esta investigación realizar un estudio de caso de una maestra para tratar de conocer cómo utiliza el huerto escolar, qué temas se trabajan con el mismo, y cuál es su finalidad. Para ellos diseñamos una serie de indicadores haciendo hincapié en aspectos sobre metodología y tratamiento de la sostenibilidad, con el fin de acercarnos a una comprensión de cómo trabaja ambos aspectos, es decir el grado de integración de la sostenibilidad en la experiencia de huerto escolar.

\section{MARCO TEÓRICO}

\subsection{Sostenibilidad y Educación para la sostenibilidad}

Según Briceño y Equipo Área Educada (2012, p.281), la sostenibilidad se refiere a las acciones que ayudan a la transformación cultural y social del ser humano a nivel global, cuyo objetivo es la superación de las actuales amenazas a la humanidad. Sin embargo, se piensa que sólo abarca la conservación y protección de los recursos naturales de la fauna y de la flora, y no es así, también lo social y lo económico, medidas políticas, tecnológicas, culturales y educativas (Zabala y García, 2009).

Según Gil y Vilches (2006), la sostenibilidad exige tomar en consideración la totalidad de problemas interconectados, lo que sólo es posible a escala planetaria.

La UNESCO caracteriza la sostenibilidad como una categoría sistémica compleja que incluye e interrelaciona los aspectos económicos, sociales, culturales y ambientales del desarrollo humano que se deben contemplar en los procesos de formación (Aznar y Ull, 2009).

"Hablar de sostenibilidad es hablar de un imperativo ético que tiene que ver con cuestiones de protección del medio natural, reducción de la pobreza, igualdad de sexos, promoción de la salud, derechos humanos, comprensión cultural y paz, producción y consumo responsables, acceso igualitario a las TIC, etc.”, tal como recoge la Declaración de la Década de la Educación para el Desarrollo Sostenible (2005-2014) (CADEP-CRUE, 2012, p.5).

Para Jiménez-Fontana, Azcárate, García-González y Navarrete (2015), estamos ante una crisis ecológica, crisis económica, de valores, crisis en la educación, de pensamiento. En definitiva, ante una crisis sistémica que cuestiona el conocimiento del mundo.

Educar para la sostenibilidad constituye un objetivo que implica un esfuerzo educativo enfocado a: cambiar los modelos interpretativos en relación con las cuestiones ambientales y ofrecer la vivencia de modelos alternativos que permitan su análisis y contraste (Junyent, Geli y Arbat, 2003).

\subsection{Ambientalización curricular}

La ambientalización curricular, concepto al que se llegó por consenso establecido por la red ACES (Ambientalización Curricular de los Estudios Superiores), es un proceso continuo de producción cultural tendente a la formación de profesionales comprometidos con la búsqueda 
permanente de las mejores relaciones posibles entre la sociedad y la naturaleza, atendiendo a los valores de la justicia, la solidaridad y la equidad, aplicando los principios éticos universalmente reconocidos y el respeto a las diversidades (Junyent et al., 2003).

Según Junyent et al. (2003), el Grupo de Trabajo Complex, dentro de esta misma red, realiza otra definición de Ambientalización curricular, entendiendo ésta como "un proceso complejo y dinámico que se edifica sobre tres pilares: la construcción de una nueva ética colectiva, un nuevo estilo de pensamiento y una nueva acción transformadora". Las 10 características que deberían formar parte de un estudio ambientalizado para esta red serían:

- Complejidad.

- Orden disciplinar: flexibilidad y permeabilidad.

- Contextualización.

- Tener en cuenta al sujeto en la construcción del conocimiento.

- Considerar los aspectos cognitivos y afectivos de las personas.

- Coherencia y reconstrucción entre teoría y práctica.

- Orientación prospectiva de escenarios alternativos.

- Adecuación metodológica.

- Generar espacios de reflexión y participación democrática.

- Compromiso para la transformación de las relaciones Sociedad-Naturaleza.

El tratamiento en el aula de líneas metodológicas de ambientalización curricular según García- González, Jiménez-Fontana y Navarrete (2017), permite caracterizar los procesos de enseñanza-aprendizaje, coherentes con la sostenibilidad. Para García-González et al. (2015), reflejar los principios de la sostenibilidad en el sistema aula como escenario de construcción fundamentada, requiere un clima de intercambio, debate y diálogo, de acción y participación y por tanto una organización metodológica, estructura y funcionamiento que ofrezcan espacios, tiempos, pautas y recursos para este fin.

\subsection{El huerto escolar}

En la búsqueda de referencias bibliográficas que nos hablen de fortalezas en relación al uso de huertos escolares como recurso educativo encontramos las siguientes:

- Eugenio y Aragón (2016, p.3), destacan:

Los huertos constituyen laboratorios vivos en los que observar y experimentar sobre sus componentes y procesos, por lo que complementan a nivel práctico los contenidos teóricos de las asignaturas de Ciencias de la Naturaleza y Educación Ambiental.

1. Su uso potencia el aprendizaje por indagación, al permitir poner en práctica habilidades y trabajar procedimientos relacionados con el método científico (Ceballos, Escobar y Vílchez, 2014).

2. Fomentan la implementación de metodologías activas y experienciales que facilitan aprendizajes significativos (Bell y Dyment, 2008).

3. Su uso tiene beneficios sobre la salud mental y emocional, y contribuye al desarrollo de una comunidad escolar más creativa, activa físicamente, pacífica y eficaz en el trabajo colaborativo (Eugenio y Aragón, 2016).

- Para Ceballos et al. (2014) la realización de un proyecto de huerto puede cubrir múltiples aspectos en la formación del alumnado. Junto con los beneficios para su desarrollo psicológico y social derivados del trabajo en equipo, son varios los objetivos principales más frecuentes: como apoyo al área de ciencias naturales, en su doble vertiente de afianzar contenidos conceptuales y desarrollar distintas habilidades relacionadas con el método científico, como parte de la educación para la salud, fomentando una alimentación saludable o como aproximación a la educación ambiental. 
Añadimos a estas fortalezas los siguientes aspectos que bajo nuestro punto de vista, y apoyándonos en la revisión bibliográfica, pueden aumentar la profundidad y complejidad en relación a lo que aportan los huertos escolares y que como tales deberían tenerse en cuenta:

- Reflexión y actuación conjunta como oportunidades en huertos escolares: En una educación por la Sostenibilidad que ayude a mirar los fenómenos con una visión sistémica, a participar en las decisiones y a actuar en un contexto comunitario, el huerto puede ser un contexto próximo que permita desarrollar la capacidad de actuar en el ámbito de la Sostenibilidad. La acción resulta demasiado central, y se puede dedicar poco tiempo a la reflexión sobre las acciones que se desarrollan (Rekondo, Espinet y Fitó, 2012, p.733). De ahí pensamos la propuesta de no perder de vista ambas.

- Formación del profesorado creciente en huertos escolares: Para poner en marcha huertos escolares es necesaria una adecuada formación del profesorado y en este sentido Eugenio, Zuazagoitia y Ruiz-González (2018), hablan del emergente movimiento de huertos educativos que se está impulsando desde algunas universidades españolas acuñando el término Huertos EcoDidácticos, incidiendo no sólo en su función educativa, sino también en las características que debe revestir su manejo.

- Huertos comunitarios generadores de cambio educativo y social: Para Conde, Borrella y Durán (2009), es de gran interés conseguir que distintas generaciones participen en los huertos escolares. En esta misma línea, Barrón y Muñoz (2015), destacan que la experiencia de Huertos escolares comunitarios es una herramienta transversal, de valor educativo y social, que permite integrar competencias tanto básicas como transversales de todo el currículo, así como trabajar valores de muy diversa índole.

- Huerto escolar para fomentar el pensamiento sistémico y la recuperación de la cultura: Para Llerena y Espinet (2017), la agroecología escolar impulsa un diálogo entre la escuela como entorno de aprendizaje y como actor. Es también punto de encuentro con saberes no científicos, locales, una recuperación de la cultura anterior olvidada.

- Decrecimiento como hecho indiscutible presente y futuro en cuestión ambiental y de huertos escolares: También se está investigando y aportando líneas para avanzar en relación con los huertos escolares y sociales como recurso para la educación en y para el decrecimiento, contribuyendo a dar una respuesta a un hecho real y no a una propuesta ideológica. Esto hace referencia al previsible agotamiento de multitud de recursos energéticos y materiales (RodriguezMarín, Fernández-Arroyo y García-Díaz, 2015). Para los mismos autores, lo que propugna la sostenibilidad parece ignorar que el decrecimiento ya es una realidad. En cualquier caso, hacer hincapié en la cuestión del decrecimiento es algo que trabajando en cuestiones de ambientalización curricular nunca deberíamos olvidar.

Aunando todas estas cuestiones en torno a los huertos escolares que acabamos de referenciar, vemos como las mismas suponen preparar a las generaciones presentes para convivir con esta realidad del decrecimiento, estando sin duda mejor capacitados para afrontarlo desde apropiadas posiciones ideológicas y competenciales. Algo que desde el huerto escolar se puede iniciar con gran facilidad, incorporando visiones esperanzadoras al poner en práctica soluciones factibles, formadoras, generadoras de cambio y relaciones en todos los sentidos, sanas y respetuosas con uno mismo, los habitantes del planeta y con el medio y hasta emocionalmente positivas como vemos cuando las ponemos en marcha con adecuada metodología.

Respecto al cómo trabajar a nivel metodológico en los huertos escolares para poder lograr el cumplimiento de los máximos objetivos: Encontramos que la investigación escolar, con propuestas constructivistas, podría ser un marco de referencia en el que transitar en este sentido. Así líneas básicas a nivel metodológico propuestas por Rivero, Martín del Pozo, Solís y Porlán (2017) tales como una metodología basada en la investigación del alumnado, partir de problemas que determinan una secuencia de actividades, y contar con el profesor como facilitador de esos procesos de investigación, han sido un claro referente en nuestra investigación. 


\section{OBJETIVOS Y PREGUNTA DE INVESTIGACIÓN}

\subsection{Objetivos de la investigación}

- Diseñar unos indicadores para analizar la práctica de huerto escolar siguiendo las categorías de ambientalización curricular presentadas por la red ACES.

- Conocer la metodología llevada a cabo en el desarrollo de la experiencia.

- Conocer en qué grado de integración se encuentra la sostenibilidad en una experiencia de huerto escolar llevado a cabo por una maestra de primaria.

\subsection{Pregunta de investigación}

¿La maestra entrevistada integra la sostenibilidad en su experiencia de huerto escolar? Si es así, ¿en qué grado y cómo contribuye a la ambientalización curricular?

\section{Metodología}

Se ha utilizado una metodología cualitativa de corte interpretativo, en la misma se realizó un análisis de análisis de contenido por los tres investigadores y en distintos momentos. Dentro de esta, hemos realizado un estudio de casos tratando de entender la manera que tienen algunos maestros de trabajar en el huerto escolar. Nos hemos desplazado a todos los colegios de la ciudad de Cáceres, ciudad con una población cercana a los 100.000 habitantes, pero aún bastante ligada al medio rural. Después de una búsqueda exhaustiva encontramos que de la totalidad, solo tres centros trabajan con huerto escolar, así que enfocamos nuestra investigación en los mismos. Para este artículo mostramos solamente los resultados de uno de los centros educativos de $3^{\circ}$ de Educación Primaria.

Hemos realizado una entrevista estructurada con 15 preguntas. (Ver Anexo). Para la elaboración de la misma nos hemos basado en las características de un estudio ambientalizado de Junyent et al. (2003) y en otros estudios como los de Bonil, Calafell, Granados, Junyent y Tarín (2012), y García-González et al. (2017). Aunque estas características de un estudio ambientalizado originalmente estaban pensadas para estudios superiores, creemos que las mismas también pueden servir como referente para otras etapas, consiguiendo adaptaciones de las mismas. Su visión más amplia y profunda, nos parece, puede ayudar mucho tanto en aspectos investigativos, como en la puesta en práctica en el aula. Se unificaron dos de las 10 categorías del estudio, considerando ambas relacionadas entre sí (adecuación metodológica y espacios de reflexión y participación democrática). En la Tabla 1 presentamos las categorías analizadas y la agrupación realizada.

TABLA 1. Categorías de integración de sostenibilidad en los centros escolares.

\begin{tabular}{|c|c|}
\hline $\begin{array}{l}\text { Análisis sobre cómo ha sido la metodología llevada a } \\
\text { cabo por el maestro en relación con la sostenibilidad } \\
\text { en el huerto escolar }\end{array}$ & $\begin{array}{c}\text { Análisis del tratamiento de temáticas de sostenibilidad } \\
\text { con una visión más global y comprometida hacia el medio } \\
\text { ambiente }\end{array}$ \\
\hline $\begin{array}{l}\text { Adecuación metodológica } \\
\text { Espacios de reflexión y participación de } \\
\text { (Preguntas } 4,5,6 \text { y } 8 \text { ) }\end{array}$ & $\begin{array}{l}\text { Compromiso para la transformación de las relaciones } \\
\text { sociedad-naturaleza (Pregunta 11) }\end{array}$ \\
\hline Flexibilidad y permeabilidad (Pregunta 7) & Complejidad. (Pregunta \\
\hline $\begin{array}{l}\text { La persona como elaboradora del } \\
\text { (Pregunta 3) }\end{array}$ & $\begin{array}{l}\text { Contextualización. Posicionamiento espacial y } \\
\text { (Pregunta 15) }\end{array}$ \\
\hline \multirow{2}{*}{$\begin{array}{l}\text { Coherencia e interacción entre teoría y práctica } \\
\text { (Preguntas } 1 \text { y 2) }\end{array}$} & $\begin{array}{l}\text { La persona considerada en todos sus aspectos (éticos y } \\
\text { estéticos) (Preguntas } 12 \text { y 13) }\end{array}$ \\
\hline & $\begin{array}{l}\text { Asunción de escenarios alternativos (Que respeten } \\
\text { generaciones futuras) (Pregunta 10) }\end{array}$ \\
\hline
\end{tabular}

Fuente: Modificado de Junyent et al. (2003) 
A la hora de analizar los resultados se proponen 5 indicadores para ver de manera más clara la metodología que se ha utilizado para el trabajo de huerto desde el papel del maestro como referente, si es más constructivista o más tradicional. Los indicadores van de menos constructivista a más, pasando por algunas fases intermedias. Sin embargo, hemos propuesto 3 indicadores para comprobar el grado de integración de la sostenibilidad en la práctica de aula con el huerto escolar desde algunos enfoques de sostenibilidad.

\subsection{Indicadores}

Los indicadores elaborados para conocer más de cerca la metodología llevada a cabo han sido revisados por dos expertos en didáctica de las ciencias experimentales. Tienen en parte como referente los indicadores de García-González et al. (2017) e indicaciones metodológicas de Rivero et al. (2017). Estos son:

1. Simple: Metodología tradicional (el maestro es el único que dirige el proceso de enseñanza aprendizaje) y el alumno tiene un papel más receptivo. No se parte de preguntas problema ni se da pie a la investigación del alumno, no se lleva a cabo evaluación de lo trabajado en torno al huerto escolar.

2. Inicial: El alumno adquiere un protagonismo solo puntual en el proceso de enseñanza-aprendizaje, pero aun así es el profesor quien lo dirige y lidera en exclusividad. No se parte de preguntas problema ni se da pie a la investigación del alumno, no se lleva a cabo evaluación de lo trabajado en torno al huerto escolar.

3. Medio: El maestro lidera el proceso y deja en varios momentos el protagonismo aunque escaso al alumno, es decir trata de implicarle en el proceso de E-A, pero de una forma menos controlada que en el caso anterior. No se parte de preguntas problema, ni se da pie a la investigación del alumno, no se lleva a cabo evaluación de lo trabajado en torno al huerto escolar.

4. Avanzado: En parte del proceso de E-A se sigue una dinámica de aula más abierta (basada a menudo en preguntas problema, dando lugar a la investigación por parte del alumnado), aunque en la estructura general de la secuencia de intervención no lo mantiene todo el tiempo. Se realiza en parte un seguimiento del proceso desde el planteamiento del problema escolar hasta su resolución, reflexionando acerca de lo aprendido.

5. Completo: El maestro se comporta en todo momento como un mediador en el proceso de enseñanza aprendizaje. La clase está abierta a todos los participantes teniendo en cuenta las ideas de los alumnos, las preguntas problema y la investigación por parte del alumnado. Se lleva a cabo una evaluación procesual, individualizada e integrada en todas las asignaturas y aspectos de la vida del centro de forma reflexiva.

Por otra parte (ver tabla I), los indicadores acerca del tratamiento de temáticas de sostenibilidad con una visión más global y comprometida hacia el medio ambiente creados a propósito para este estudio por nosotros, serían en este caso:

1. Escasa: Se trabaja nada o casi nada.

2. Intermedia: Se incorpora ese objetivo bastante, pero no lo suficiente.

3. Completa: Lo trabaja todo, o trabaja uno de los puntos clave en profundidad.

Se decidió analizar este último indicador con tres grados, dado que los resultados obtenidos no aportaban más información al respecto, por lo que podíamos llegar a saber si se trabajaba más o menos, pero no cómo se trabajaba.

En la tabla 2, se explica en qué nos hemos basado para analizar los resultados. En la primera columna, aparecen las categorías de ambientalización curricular. Cada una de ellas se relaciona con un objetivo. En la tercera columna aparecen los indicadores vistos anteriormente. 
TABLA 2. Categorías de sostenibilidad y objetivo a cumplir con los indicadores correspondientes

\begin{tabular}{|c|c|c|c|c|c|c|c|}
\hline \multirow[b]{2}{*}{ Categoría } & \multirow{2}{*}{$\begin{array}{c}\text { Objetivo deseable a } \\
\text { comprobar si se cumple desde } \\
\text { el papel del maestro }\end{array}$} & \multicolumn{6}{|c|}{ Resultados } \\
\hline & & Simple & Inicial & $\begin{array}{c}\text { Medi } \\
\text { o }\end{array}$ & \multicolumn{2}{|c|}{ Avanzado } & Completo \\
\hline $\begin{array}{l}\text { Adecuación metodológica } \\
\text { y espacios de reflexión y } \\
\text { participación democrática }\end{array}$ & $\begin{array}{c}\text { Trabajar de manera colaborativa } \\
\text { evaluando de manera procesual } \\
\text { y utilizando una metodología de } \\
\text { investigación escolar } \\
\text { participativa que fomente la } \\
\text { reflexión creando espacios que } \\
\text { impliquen a todos los colectivos } \\
\text { para promover un futuro } \\
\text { sostenible }\end{array}$ & 1 & 2 & 3 & & 4 & 5 \\
\hline $\begin{array}{l}\text { Flexibilidad } \\
\text { permeabilidad }\end{array}$ & $\begin{array}{l}\text { Relacionar el huerto con otras } \\
\text { materias (matemáticas, lengua, } \\
\text { música...) }\end{array}$ & 1 & 2 & 3 & & 4 & 5 \\
\hline $\begin{array}{|lr|}\text { La persona } & \text { como } \\
\text { elaboradora } & \text { del } \\
\text { conocimiento } & \end{array}$ & $\begin{array}{l}\text { Reflexionar sobre el trabajo } \\
\text { realizado y comprobar que son } \\
\text { conscientes del por qué }\end{array}$ & 1 & 2 & 3 & & 4 & 5 \\
\hline $\begin{array}{l}\text { Coherencia e interacción } \\
\text { entre teoría y practica }\end{array}$ & $\begin{array}{c}\text { Llevar a la práctica todo el } \\
\text { trabajo de huerto utilizando una } \\
\text { metodología coherente de forma } \\
\text { procesual e investigativa de } \\
\text { inicio a fin }\end{array}$ & 1 & 2 & 3 & & 4 & 5 \\
\hline \multirow{2}{*}{ Categoría } & \multirow{2}{*}{$\begin{array}{c}\text { Objetivo deseable a } \\
\text { comprobar si se cumple desde } \\
\text { algunos enfoques más globales } \\
\text { de la sostenibilidad }\end{array}$} & \multicolumn{6}{|c|}{ Resultados } \\
\hline & & \multicolumn{2}{|c|}{ Escaso } & \multicolumn{2}{|l|}{ Medio } & \multicolumn{2}{|c|}{ Completo } \\
\hline $\begin{array}{l}\text { Compromiso para } \\
\text { transformar las relaciones } \\
\text { sociedad- naturaleza }\end{array}$ & $\begin{array}{l}\text { Trabajar valores de cuidado con } \\
\text { el medio y ponerlos en práctica }\end{array}$ & 1 & & \multicolumn{2}{|l|}{2} & \multicolumn{2}{|r|}{3} \\
\hline Complejidad & $\begin{array}{l}\text { Utilizar el huerto para mostrar } \\
\text { que todo lo que nos rodea está } \\
\text { interrelacionado. (Alimentación, } \\
\text { pobreza, contaminación, } \\
\text { economía...) }\end{array}$ & 1 & & \multicolumn{2}{|l|}{2} & \multicolumn{2}{|r|}{3} \\
\hline $\begin{array}{l}\text { Contextualización. } \\
\text { Posicionamiento espacial y } \\
\text { temporal }\end{array}$ & $\begin{array}{c}\text { Relacionar el huerto con } \\
\text { problemas, locales, globales del } \\
\text { presente y futuro }\end{array}$ & \multicolumn{2}{|l|}{1} & \multicolumn{2}{|l|}{2} & \multicolumn{2}{|r|}{3} \\
\hline $\begin{array}{l}\text { La persona considerada en } \\
\text { todos sus aspectos (éticos y } \\
\text { estéticos) }\end{array}$ & $\begin{array}{c}\text { Tener en cuenta las emociones e } \\
\text { ideas de los alumnos }\end{array}$ & 1 & \multicolumn{3}{|c|}{2} & \multicolumn{2}{|r|}{3} \\
\hline $\begin{array}{l}\text { Asunción de escenarios } \\
\text { alternativos (que respeten } \\
\text { generaciones futuras) }\end{array}$ & $\begin{array}{c}\text { Plantear a los alumnos distintos } \\
\text { escenarios de la problemática } \\
\text { ambiental para considerar } \\
\text { repercusiones }\end{array}$ & \multicolumn{2}{|l|}{1} & \multicolumn{2}{|l|}{2} & \multicolumn{2}{|r|}{3} \\
\hline
\end{tabular}

Codificación. CAP-Colegio “AP”. (por ejemplo CAP2, CAP pregunta 2 de la entrevista) 


\section{RESULTADOS. ANÁLISIS Y DISCUSIÓN}

Comenzamos a exponer los resultados en las categorías directamente relacionadas con la metodología llevada a cabo para trabajar en el huerto escolar. Los indicadores establecidos en esta investigación para este caso iban de 1 a 5 (simple, inicial, medio, avanzado, completo), siguiendo la dirección desde una metodología más tradicional a otra más constructivista.

En relación con la categoría de adecuación metodológica y espacios de reflexión y participación democrática los resultados obtenidos nos muestran lo siguiente:

C1AP4: "Nosotros aquí trabajamos de manera constructivista. Este centro trabaja por proyectos desde hace varios años $y$, aunque no es lo común, nunca hago examen en ninguna asignatura. Yo todo lo tengo con rúbricas y gráficas. Todo lo tengo registrado, es la mejor manera de organizarme y de ver el avance del alumno de manera más clara y justa”.

C1AP5: "En el huerto trabajamos por equipos, cada equipo se ocupa de una tarea ya sea medir, quitar malas hierbas, sembrar, regar, recolectar... Cada grupo va rotando para poder realizar todo tipo de tareas de huertos. Previamente ya saben lo que van a hacer así que investigan acerca de los cuidados y la mejor manera de realizarlo".

C1AP6: "Creo que para que un trabajo esté bien hecho siempre necesita de una planificación previa. Yo necesito tenerlo todo planeado antes y así veo la única manera de que salgan las cosas medio decentes".

C1AP8: "El huerto como tal no se evalúa sino que lo tengo en cuenta en las dos materias. Jamás hago exámenes y sorprendentemente son ellos mismos los que se evalúan, cuando la planta sembrada no crece o se seca, los deberes son investigar posibles errores que hayamos cometido, o conocer que ha podido pasar, si es el suelo, el riego, el sol... por descarte vamos sacando entre todos el problema y es una manera de evaluar el trabajo hecho".

Como hemos podido ver en estos fragmentos de la entrevista, en este colegio, los resultados muestran a priori con respecto a la adecuación metodológica y espacios de reflexión y participación democrática que se trabaja de manera cooperativa evaluando de manera procesual y utilizando una metodología participativa que fomenta la reflexión como bien diría (García-González y otros, 2017). La maestra se comporta como un mediador en el proceso de enseñanza aprendizaje. La clase está abierta a todos los participantes teniendo en cuenta las preguntas problema, ideas de los alumnos y la investigación por parte de los mismos. Se utilizan diferentes recursos para trabajar y evaluar todo el proceso en relación con el huerto escolar por lo que estaríamos en torno al indicador 5 (completo).

Continuamos con la categoría de flexibilidad y permeabilidad.

C1AP7: "El huerto está integrado en la asignatura de lengua y matemáticas".

Dado que la maestra integra el proyecto de huerto en otras asignaturas con un método de trabajo activo, que permite la participación del alumnado, basado en la formulación de preguntas y la búsqueda de las respuestas, realizando pruebas con las plantas del huerto, etc, y teniendo en cuenta a los alumnos, estaríamos ante el indicador 5.

Respecto a la categoría: "La persona como elaboradora del conocimiento":

C1AP: "Al trabajar con grupos investigadores, toda la información la traían ellos ya sabida y ellos mismos reflexionaban sobre lo que se había hecho bien y lo que no. Es verdad que no teníamos un espacio ni momento concreto para hablar sobre eso, sino que sobre la marcha del día a día se iba tratando". 
Nos encontraríamos en el indicador 4 (avanzado) ya que se sitúa al alumno ante una investigación que el maestro guía y deja en varios momentos el protagonismo al alumno porque son estos los que exponen sus hallazgos y reflexiones, es decir, trata de implicarles en el proceso de EA, pero el seguimiento se realiza de manera parcial.

Continuamos con la categoría de coherencia e interacción entre teoría y práctica:

C1AP1: "En mi caso, he utilizado el huerto como complemento en asignaturas como lengua y matemáticas".

"En el caso de lengua se utilizó para crear textos escritos, entrevistas y tipos de palabras como son los verbos, sustantivos y adjetivos".

"En matemáticos vimos las dimensiones, las medidas, dividir surcos..."

C1AP1: "En lengua creamos un grupo de investigadores en el cual debían investigar acerca de qué sembrar, cuando, cuidados, características de la planta. Para trabajar la entrevista hicimos una entrevista a través de Skype con un experto que trabaja en un vivero, para organizarnos y saber qué necesitamos para trabajar en el huerto creamos una lisa de instrumentos, otra lista verbos que informan sobre el trabajo de huerto... También, realizamos una carta para pedir ayuda y agradecimiento a Pedro, el conserje".

"En el caso de las mates fuimos con un metro y medimos el perímetro, una vez calculadas las medidas calculamos donde debían ir los surcos... Fue un trabajo muy enriquecedor tanto para ellos como para mí".

C1AP2: "Llevo a cabo los contenidos de manera práctica en el huerto, "in situ"”.

"Por ejemplo, para que sean ellos los que investiguen acerca de lo que van trabajando en el huerto, los propios alumnos son los encargados de observar toda la evolución. Un caso curioso es que quisieron sembrar garbanzos ya que conocían de antes su germinación, debido a que cursos anteriores habían hecho este experimento con un yogur. Al ser petición de ellos lo sembraron y vieron que no salía nada. Yo como maestra pedí a cada uno que investigara en casa cual era el motivo por el que no salían, y llegaron a la conclusión de que fue debido a que no era la época del año adecuada para su plantación".

Estaríamos en el indicador 4 (avanzado). En parte del proceso de E-A se sigue una dinámica de aula más abierta con preguntas problema tal como la cuestión de germinación, donde también se tienen en cuenta sus ideas previas al respecto, aunque en la estructura general no lo mantiene todo el tiempo. Vemos como en lengua y matemáticas utiliza contenidos conceptuales y procedimentales para aplicar directamente en esas asignaturas, haciendo consciente al alumnado de todo lo aprendido, y relacionando así todos los contenidos de una forma más global.

A continuación mostramos los resultados de categorías en las que la pretensión no era analizar tanto la metodología seguida por la maestra, como el grado de integración de cuestiones más globales y con mayor nivel de compromiso hacia el medio ambiente en relación con la sostenibilidad en el aula y en el huerto escolar. Para estas categorías hemos utilizado indicadores desarrollados en tres niveles de integración de la sostenibilidad (escaso, intermedio, completo).

Con respecto a la complejidad, para buscar si existen adaptaciones iniciales que la integren en primaria, tenemos la pregunta 9.

C1AP9: "Son alumnos son muy pequeños de 7 y 8 años y la verdad es que nosotros lo relacionamos directamente con la alimentación e higiene. Bueno, aunque ahora que lo dices también con el consumo de agua".

"Nosotros no utilizamos ningún tipo de pesticidas. En septiembre cuando arranca el huerto echamos abono de vaca que me facilita mi padre y es lo único que hacemos". 
Nos encontraríamos en el indicador 2 (intermedio) ya que no lo interrelaciona todo, pero sí algo, como por ejemplo la alimentación saludable, la no utilización de productos químicos...

Con respecto a la categoría de contextualización y relación del huerto con otros problemas tanto del presente o del futuro (pregunta 15) la respuesta obtenida fue que admitió no relacionar el huerto con otros problemas locales, globales del presente y futuro, por lo que nos encontraríamos en el indicador 1 (escasa).

En la siguiente categoría, la persona considerada en todos sus aspectos:

C1AP13: "Lo de los alumnos es algo increíble, están súper motivados hasta el punto de quedarse sin recreo para estar allí. Todo lo que sea pringarse de agua y tierra les encanta y los resultados de este trabajo son inmejorables".

Nos encontraríamos en el indicador 2 (intermedio). Se cumple el objetivo de manera parcial, ya que no hace que el alumnado exprese de forma explícita sus ideas y emociones respecto al tema y sea consciente de ellas y de su evolución con el trabajo sobre el huerto escolar, pero sí hace una observación o seguimiento de todo esto, lo que le sirve como un aspecto de evaluación del proceso de E-A llevado a cabo.

Para finalizar este análisis tenemos la categoría de asunción de escenarios alternativos.

C1AP10: "La verdad es que no, aunque bueno,... si se considera el motivarles a comer verduras sanas y de temporada, siempre por ejemplo el ir los miércoles al mercado, y comprar allí la fruta y verdura en vez de en los grandes hipermercados donde te encuentras de todo y durante todo el año".

Creemos que para esta categoría nos encontraríamos ante el indicador 1 (escaso), ya que admite no integrar la sostenibilidad en apenas ningún escenario alternativo fuera del huerto.

Si continuamos este análisis descriptivo, la siguiente categoría sería con respecto al compromiso para la transformación de las relaciones sociedad-naturaleza,

C1AP11: "Creo que trabajar el huerto en los colegios es más complicado de lo que se cree. Son muchas cosas a tener en cuenta y mucho trabajo tanto por parte de los alumnos como por mi parte, entonces creo que aunque no sea todo lo completo que debería ser este trabajo, con el granito de arena que aportamos estamos en compromiso con la naturaleza aunque no nos quedamos ahí, porque con todo lo que aprendemos en clase, si lo llevan a cabo en casa ya están realizando un gran trabajo".

Nos encontraríamos, con todas las dificultades por los matices que conlleva analizar esta categoría, ante lo que nos parece podría ser el nivel 2 (intermedio). Se incorpora el objetivo (trabajar valores de cuidado con el medio y ponerlos en práctica) bastante, en algunas temas como alimentación, higiene y consumo de agua; sin embargo, no profundizan en aspectos como la contaminación que causa el traer los alimentos desde grandes distancias, el empaquetado de los productos, el desarrollo local, la conservación de semillas autóctonas, los huertos de su entorno... Entendemos que como dice la maestra son muchas cosas a tener en cuenta y mucho trabajo, y especialmente queremos destacar todo lo que sí se ha conseguido y que aplicándolo en su hogar ese grano de arena puede ir haciendo montañas realmente con el compromiso de todos.

A continuación, en la figura 1, presentamos un resumen de las categorías analizadas, indicadores y resultados obtenidos, que de forma gráfica pueden servir para ver con mayor claridad donde están los puntos fuertes y débiles del trabajo de integración de la sostenibilidad. 
FIGURA 1. Rueda de categorías de sostenibilidad con resultados según indicadores alcanzados C1AP

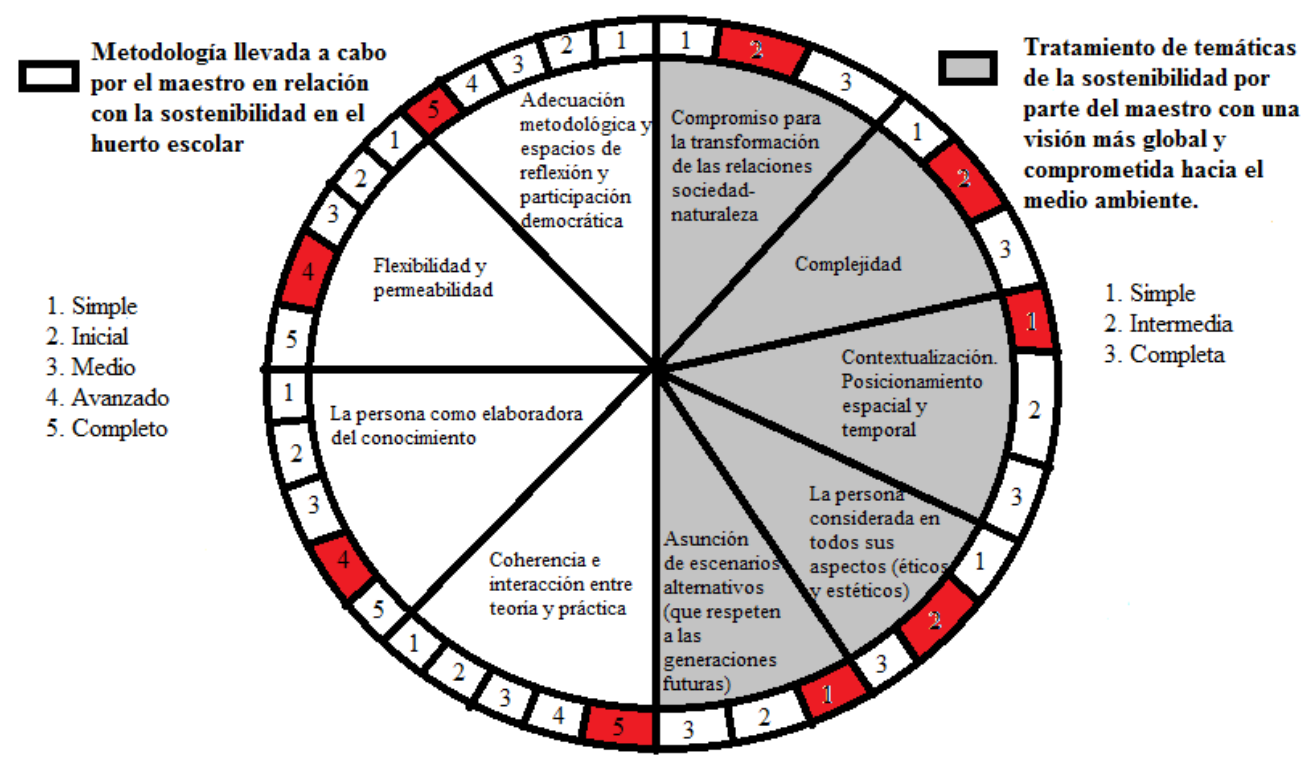

Fuente: elaboración propia

\section{Conclusiones}

Respecto al primer objetivo de la investigación (Diseñar unos indicadores para analizar la práctica de huerto escolar siguiendo las categorías de ambientalización curricular presentadas por la red ACES), creemos que las categorías y los indicadores elaborados, han servido para analizar el proceso de E-A llevado a cabo, y comprender si se ha integrado la sostenibilidad y el papel de la maestra en ello.

Respecto al segundo objetivo (Conocer la metodología llevada a cabo en el desarrollo de la experiencia), hemos comprobado que la misma tiene un enfoque cercano al constructivismo en bastantes aspectos. Trabaja por proyectos de investigación escolar haciendo al alumnado protagonista del proceso de E-A, permitiendo que los alumnos se planteen cuestiones a resolver, e indagando sobre ellas partiendo de sus ideas previas. Reflexionan acerca de los temas trabajados y sobre los posibles errores cometidos. La maestra integra muchas cuestiones conceptuales y procedimentales en relación al huerto escolar en las distintas materias.

Los alumnos adquieren la responsabilidad sobre el propio trabajo encargándose de los diferentes enfoques tanto de cuidado, como de estudio del huerto. Realiza una evaluación global a través de rúbricas teniendo en cuenta todo el proceso de E-A llevado a cabo.

Para algunos enfoques más globales sobre sostenibilidad encontramos que en el aula existe una visión sostenible con respecto a la alimentación, el agua, la higiene, la utilización de productos ecológicos para el cuidado del huerto... pero no se ve de forma más explícita la interrelación entre todas estas cuestiones del aula y la responsabilidad del hombre en ello, por ejemplo no se tratan temas como el hiperconsumo, los pesticidas, el consumo de productos lejanos, no de temporada, el hambre, la soberanía alimentaria... lo que se acercaría más a lo que se refiere la complejidad en sus aspectos: sistémicos, hologramáticos y dialógicos (Bonil et al., 2010). Entender que "el todo" forma parte de cada uno de nosotros, y que las acciones que hagamos sobre el medio volverán como consecuencias, debería ser más trabajado. Al igual que tratar la conexión naturaleza, cultura y sociedad es esencial para que el alumno se encuentre a sí mismo y encuentre sentido a todo lo que le rodea. 
Esta experiencia muestra la dificultad para integrar todas las temáticas deseables en relación con la sostenibilidad. Facetas como plantear escenarios alternativos para ampliar el campo de visión del alumnado no son abordados. Tampoco se aborda la contextualización, el pasado, presente y futuro en relación con estas temáticas, lo que no resta mérito a todo lo desarrollado, pero vemos que estos retos siguen pendientes. Quizá un tratamiento integrado de estas cuestiones en todas las materias, si somos conscientes de su importancia, podría ayudar a dejar espacios de trabajo y reflexión para lo que no se trabaja lo suficiente. El trabajo colaborativo es cada vez más necesario para llegar a logros como estos de mayor alcance.

Respecto a las actitudes y emociones del alumnado en el huerto, la maestra comenta el entusiasmo e implicación del alumnado.

En relación al último de los objetivos de la investigación (Conocer en qué grado de integración se encuentra la sostenibilidad), observamos la existencia de una gran coherencia entre el marco teórico con lo que la maestra pone en práctica. Quedaría pendiente abordar otras cuestiones sobre sostenibilidad, pero lo tratado se lleva a cabo en la misma línea que las investigaciones y experiencias referenciadas en el trabajo.

La falta de tiempo, la saturación y la escasa motivación de algunos maestros hace más difícil actividades como la del huerto escolar que requieren tiempo extraordinario en horario no lectivo. También la falta de formación y el no conseguir que sean experiencias compartidas por varios maestros es un obstáculo a la hora de trabajar la sostenibilidad. No se puede enseñar a solucionar un problema si no se conoce a fondo el problema, o cómo encontrar oportunidades de plantearlo integrado en todas las asignaturas, ni tampoco pretender que sólo trabajándolo en una asignatura quede integrado en todas las dimensiones deseadas.

El huerto escolar debería abordar más aspectos en relación con la ambientalización ( currricular, de la vida del centro, de la relación con la familia, la sociedad, del comedor escolar,...) es un reto a nuestro alcance según se vayan consolidando estas experiencias.

En definitiva, el huerto escolar, trabajado en la medida de lo posible teniendo en cuenta visiones más amplias (tal y como nos ofrecen las categorías de ambientalización curricular vistas), supone una gran oportunidad para lograr la ambientalización curricular y del centro, abriendo una mayor concienciación e implicación ambiental no solo en la vida personal y del centro, sino en relación al entorno del mismo y a la sociedad en general. Los huertos escolares son semillas de cambio. En tiempos de crisis ambiental como esta, desde los centros educativos de todos los niveles podemos ofrecer también nuestras soluciones reales. Estas nos pueden hacer ver que otro mundo es posible ya, mientras vamos sembrando en comunidad.

\section{Referencias}

Aznar, P. y U1l, M.A. (2009). La formación de competencias básicas para el desarrollo sostenible: el papel de la Universidad. Revista de Educación, no extraordinario, 219-237.

Barrón, Á. y Muñoz, J.M. (2015). Los huertos escolares comunitarios: fraguando espacios socioeducativos en y para la sostenibilidad. Foro de Educación, 13(19), 213-239.

Bell, A.C. y Dyment, J.E. (2008). Grounds for health: the intersection of green school grounds and health-promoting schools. Environmental Education Research, 14(1), 77-90.

Bonil, J., Calafell, G., Granados, J., Junyent, M. y Tarín, R.M. (2012). Un modelo formativo para avanzar en la ambientalización curricular. Profesorado. Revista de Currículum y Formación de Profesorado, 2(16), 146-173.

Bonil, J., Junyent, M. y Pujol, R.M. (2010). Educación para la sostenibilidad desde la perspectiva de la complejidad. Revista Eureka sobre Enseñanza y Divulgación de las Ciencias, 7 (n extraordinario), 198-215. 
Briceño, J.J. y Equipo Área Educada. (2012). Retos para la sostenibilidad en el Área metropolitana del Valle de Aburrá de Antioquia, Colombia. Percepciones acerca de lo que entendemos por este término y ejemplos de actividades educativas. Revista Eureka sobre Enseñanza y Divulgación de las Ciencias, 9(2), 278-293.

Cadep-Crue (2012). Directrices para la introducción de la sostenibilidad en el curriculum. Actualización de la declaración institucional de 2005. Recuperado de http://docplayer.es/16105151-Directrices-para-la-introduccion-de-la-sostenibilidad-en-elcurriculum-crue.html.

Ceballos, M., Escobar, T. y Vílchez, J.E. (2014). El huerto escolar: percepción de futuros maestros sobre su utilidad didáctica. En ÁPICE (Comp.), 26 Encuentros de Didáctica de las Ciencias Experimentales (pp. 285-292). Huelva: Universidad de Huelva.

Conde, M.C., Borella, F. y Durán G. (2009). Una experiencia de huerto escolar enmarcada en huertos de ocio intergeneracionales. Aula de Innovación Educativa, 183, 44-47.

Escutia, M. (2009). El huerto escolar ecológico. Barcelona: Graó.

Eugenio, M. y Aragón, L. (2016). Experiencias en torno al huerto ecológico como recurso didáctico y contexto de aprendizaje en la formación inicial de maestros de Infantil. Revista Eureka Sobre Enseñanza y Divulgación de las Ciencias, 13(3), 667-679.

Eugenio, M., Zuazagoitia, D. y Ruiz-González, A. (2018). Huertos EcoDidácticos y Educación para la Sostenibilidad. Experiencias educativas para el desarrollo de competencias del profesorado en formación inicial. Revista Eureka sobre Enseñanza y Divulgación de las Ciencias, 15(1), 1501-1515.

García-González, E., Jiménez-Fontana, R. y Navarrete, A. (2017). Educar para la sostenibilidad desde las Ciencias Económicas. Revista Iberoamericana de Educación, 73, 155-178.

García-González, E., Jiménez-Fontana, R., Navarrete, A. y Azcárate, P. (2015). La metodología docente como estrategia para promover la sostenibilidad en las aulas universitarias. Un estudio de caso en la Universidad de Cádiz. Foro de Educación, 13(19), 85-124.

Gil, D. y Vilches, A. (2006). Algunos obstáculos e incomprensiones en torno a la sostenibilidad. Revista Eureka sobre Enseñanza y Divulgación de las Ciencias, 3(3), 507-516.

Jiménez-Fontana, R., Azcárate, P., García-González, E. y Navarrete, A. (2015). Dimensión ética de la sostenibilidad curricular en el sistema de evaluación de las aulas universitarias. El caso de la enseñanza aprendizaje de las Ciencias. Revista Eureka sobre Enseñanza y Divulgación de las Ciencias, 12(3), 536-549.

Junyent, M., Geli, A. y Arbat, E. (2003). Ambientalización curricular de los estudios superiores. Girona: Universidad de Girona.

Llerena, G. y Espinet, M. (2017). Agroecología escolar. Barcelona: Polen.

Recondo, M., Espinet, M. y Fitó, A. (2012). Las asambleas de huerto como herramienta para trabajar la toma de decisiones en Educación para la Sostenibilidad. En ÁPICE (Comp.), 25 Encuentros de Didáctica de las Ciencias Experimentales (pp. 733-740). Santiago de Compostela: Universidad de Santiago de Compostela.

Rivero, A., Martín del Pozo, R., Solís, E. y Porlán, R. (2017). Didáctica de las Ciencias Experimentales en Educación Primaria. Madrid: Síntesis.

Rodríguez-Marín, F., Fernández-Arroyo, J. y García-Díaz, J.E. (2015). El huerto escolar ecológico como herramienta para la educación en y para el decrecimiento. Investigación en la escuela, $86,35-48$.

Zabala, G. y García, T.M. (2009). La Educación del Siglo XXI de acuerdo a la perspectiva del paradigma ecológico: Una Alternativa para la sostenibilidad. Revista de Investigación, 33(68), 233-250. 


\section{Anexo}

\section{Instrumento de recogida de datos}

I. ¿Cuáles son los contenidos que trabajas a través del huerto escolar?

II. ¿Cómo llevas a cabo todos esos contenidos? ¿Cómo es la secuencia que realizas desde el origen hasta el punto final?

III. ¿Cómo te organizas con los alumnos? ¿Se reflexiona sobre lo aprendido cada vez que se trabaja en el huerto?

IV. ¿Qué metodología crees que utilizas para trabajar en el huerto?

V. ¿Cómo trabajáis en el propio huerto? ¿Qué recursos utilizas? ¿Qué dificultades se presentan a lo largo de todo el proceso?

VI. Con respecto a la planificación del trabajo en el huerto: ¿está programado con anterioridad? ¿Se cumple lo planificado a la hora de ponerlo en práctica?

VII. Cuando trabajas el huerto, ¿lo relacionas con temáticas de otras materias?

VIII. A la hora de la evaluación sobre todo lo trabajado en relación al huerto escolar... ¿Cómo la llevas a cabo? ¿Qué instrumentos utilizas?

IX. ¿Relacionas el huerto con, por ejemplo la agricultura mundial, el hambre, el hiperconsumo, pesticidas y otros problemas socioambientales? Si no es así, ¿Estarías dispuesta a incluirlos?

X. ¿Planteas escenarios futuros a los alumnos para que visualicen la repercusión y la importancia de trabajar en el huerto?

XI. ¿Crees que con el trabajo que estás realizando en el huerto estás en compromiso con la naturaleza?

XII. ¿Hay algo del huerto escolar que te cause algún interés especial y que lo estés trabajando con los alumnos de manera más profunda?

XIII. ¿Qué actitudes y emociones tienen los alumnos cuando trabajan en el huerto?

XIV. ¿Qué fortalezas y debilidades encuentras a la hora de trabajar en el huerto?

XV. Con respecto a otros aspectos fuera de lo que son los alumnos, ¿Qué oportunidades te ofrece el centro, la localidad... con respecto al huerto? ¿y qué amenazas?

XVI. ¿Hay algo que quieras aportar que no se haya dicho en la entrevista?

\section{CÓMO CITAR ESTE ARTÍCULO}

Conde Núñez, M. C., Mariscal Díaz, P. y Sánchez Cepeda, J. M. (2018). La metodología en el trabajo de huerto escolar y coherencia con la ambientalización curricular. Análisis de una práctica docente. Didáctica de las ciencias experimentales y sociales, 35, 113-126. DOI: 10.7203/DCES.35.12799 